\title{
ENSEÑANZA DEL DISEÑO PROYECTUAL EN EL TRABAJO FINAL DE CARRERA EN LA UP 'A' DE LA FAU-UNNE
}

FOGAR, Sandra R.

sanfogar@outllook.com

Profesora jefa de Trabajos Prácticos de Trabajo Final de Carrera, Unidad Pedagógica 'A'. Universidad Nacional del Nordeste, Facultad de Arquitectura y Urbanismo.

Palabras Clave: Enseñanza, estrategias didácticas, proceso de diseño proyectual. Keywords: Teaching, didactic strategies, diseño proyectual process.

\section{RESUMEN}

Se presentan avances de la investigación Estrategias didácticas para la enseñanza del Diseño Proyectual en Trabajo Final de Carrera de la FAUUNNE. ${ }^{1}$ Se abordan estrategias de enseñanza del diseño proyectual y sus vinculaciones con teorías del diseño arquitectónico y se asume la perspectiva crítica del conocimiento y la enseñanza. La reflexión sobre la enseñanza del diseño arquitectónico tensiona las prácticas instaladas por fuerza de la tradición en la facultad y las interpela en clave social, hacia la búsqueda de espacios de diálogo entre los saberes docentes y las trayectorias socioeducativas de los estudiantes.

\begin{abstract}
Advances of the investigation "Didactic Strategies for the teaching of the Diseño Proyectual in the subject Trabajo Final de Carrera of the FAU-UNNE are presented here from the approach of the teaching strategies of the diseño proyectual and his entails with theories of the architectural design and is assumed the critical perspective of the knowledge and teaching. The reflection on the education of the architectural design stresses the practices installed by force of the tradition in the faculty and addresses to them in social key, towards the search of spaces of dialog between the teacher's knowledge and the socio educational paths of the students.
\end{abstract}




\section{OBJETIVOS}

\section{Generales}

- Producir conocimientos acerca de la enseñanza del diseño proyectual en arquitectura.

\section{Específicos}

- Indagar las estrategias didácticas utilizadas para la enseñanza del diseño proyectual en el Taller Trabajo Final de Carrera de la Unidad Pedagógica "A", y proponer estrategias didácticas adecuadas para la enseñanza de ese objeto.

- Analizar las relaciones entre las condiciones socioinstitucionales, las prácticas de enseñanza y las trayectorias de los estudiantes.

\section{PLANTEO INTRODUCTORIO DEL PROBLEMA}

El trabajo tiene carácter exploratorio, enfoque que admite la redefinición del diseño, de acuerdo con la información que se va relevando y con los significados y categorías que se van construyendo. La investigación sigue una lógica cuanti-cualitativa. Como instrumentos se utilizaron encuestas a estudiantes que cursan el Taller TFC en la Unidad Pedagógica "A" y entrevista semiestructurada a docentes actuales y a exmiembros de los equipos docentes de Arquitectura $\vee$ (actualmente TFC) y de asignaturas del área del diseño.

\footnotetext{
1 Secretaría General de Ciencia y Técnica, UNNE, PI N.0138/07.

2 SILVA, CARLOS ALBERTO Y FOGAR, MARIELA DEL CARMEN (2013). Los estudiantes y su formación Escenarios y tensiones en la educación técnica. Estudio de casos del Profesorado en Tecnología del INTE y P. En: Actas de Primeras Jornadas Internacionales sobre Conflictos y Problemáticas Sociales y Terceras Jornadas Interdisciplinarias sobre Conflictos y Problemáticas Sociales en la Región del Gran Chaco. UNNE, Facultad de Humanidades, 2, 3 y 4 de mayo de 2013. Publicación en prensa en Reviste Theomai. Estudios Críticos sobre Sociedad y Desarrollo. Considerada "Revista Destacada" por los investigadores del CONICET (Encuesta de revistas en Ciencias Sociales, CONICET-Centro Redes).
}

El abordaje de la enseñanza del diseño arquitectónico exige considerar dos campos del conocimiento: el diseño arquitectónico y las prácticas de enseñanza. Ambos campos de desempeño profesional (del arquitecto y del docente) suponen un posicionamiento epistemológico, desde el cual pensar y actuar. Así pues, la investigación se apoya en los aportes de las escuelas de arquitectura del Bauhaus, Ulm y las experiencias de enseñanza de KAHN, así como en la perspectiva crítica de la educación, que al postular el conocimiento como construcción social, posibilita reflexionar sobre los aspectos sociales que operan en la enseñanza y en el aprendizaje, y reconoce el carácter histórico de la producción de conocimientos en la institución. En este sentido, resulta relevante la noción de trayectorias estudiantiles. Como plantean FOGAR y SILVA (2013): "Diversos estudios (Terigi, 2007; Glutz, 2006; Kaplan y Fainsod, 2006; Fogar y otros, 2010) muestran que las trayectorias formativas de los estudiantes se construyen en las relaciones entre competencias personales, condiciones institucionales, logros académicos, calificaciones obtenidas y respuestas institucionales a las necesidades socioeducativas. A partir de las experiencias individuales y colectivas vividas en esas trayectorias, se construye el desempeño... en su complejidad". 2

Los estudios sobre la enseñanza del diseño en el taller de arquitectura (en adelante TA) aportaron tanto a los métodos de enseñanza como a la práctica del diseño y a la formulación de interrogantes sobre qué hacen maestros y estudiantes del TA. Estas cuestiones interesan además para la enseñanza de las ciencias en que se funda el saber proyectual y la apropiación de métodos y herramientas útiles para el diseño arquitectónico.

La construcción de los conocimientos relativos a este campo requiere que los estudiantes se apropien de los diversos métodos de diseño. Como advierten los constructivistas, no hay construcción posible de la nada; pero para conocer no alcanza, sin embargo, con la adquisición de nociones teóricas, aun cuando hayan sido elaboradas por destacados teóricos de la 
"Si bien todo objeto de conocimiento es complejo, a la complejidad del diseño arquitectónico se le suma la dificultad para ubicarlo en un campo particular del conocimiento sin encorsetarlo o borrar sus límites. "

disciplina. Esto es así, fundamentalmente, porque la realidad -incluidas las representaciones ${ }^{3}$ y las vivencias del espacio- es diversa y cambiante.

Por otra parte, aun cuando hoy prime el reconocimiento de que teoría y práctica son dos realidades que operan conjuntamente, el qué y cómo enseñar la práctica de diseño proyectual sigue siendo un problema definible, en términos del dinamismo histórico. Si bien todo objeto de conocimiento es complejo, a la complejidad del diseño arquitectónico se le suma la dificultad para ubicarlo en un campo particular del conocimiento sin encorsetarlo o borrar sus límites. El diseño, su método y proceso aún no han podido alcanzar un estatus definido, como las ciencias, las artes o la tecnología, campos de los que se nutre.

Al respecto, JONAS4 expresa que "El diseño no es la tecnología porque trata con criterios borrosos y disgregados antes que con criterios objetivos, aunque... comparte muchos objetivos funcionales..., no es la ciencia porque no ofrece nuevos modelos explicativos de la realidad, pero cambia la realidad más o menos resueltamente, y más todavía el proceso experimental de investigación se parece al proceso de diseño. Obviamente, el diseño es algo muy especial".

\section{LA ENSEÑANZA DEL DISEÑO EN EL TFC. CARACTERISTICAS DE LA ASIGNATURA}

La asignatura TFC constituye un espacio de aprendizaje fundamental para la formación de los estudiantes. En ella se persigue la apropiación de aprendizajes para la construcción de una metodología de diseño arquitectónico; es, además, el último taller de la carrera en que los estudiantes ejercitan la actividad proyectual.

Son muchos los obstáculos con que los docentes nos encontramos para lograr los objetivos propuestos. El desafío es, pues, comprender tanto los problemas como las situaciones que los originan, para diseñar estrategias de enseñanza adecuadas. De aquí la necesidad de identificar indicadores del problema en una cátedra que se desarrolla con la modalidad de taller y requiere, por parte de los estudiantes, conocimientos provenientes de los distintos campos disciplinares que conforman las áreas contempladas en el plan de estudios. Requiere asimismo la capacidad para recuperar los aprendizajes construidos en los talleres anteriores.

De entre los indicadores se distinguen los propios de la enseñanza y los propios del aprendizaje, asumiendo que en la práctica operan en estrecha interdependencia. Para el análisis de los indicadores, se parte de la consideración de situaciones de enseñanza, a la luz de diferentes autores que desde la corriente crítica permiten desnaturalizar las prácticas y repensar la función de la enseñanza en la universidad. Se alude así a problemas tales como la dependencia teoría-práctica en su innegable relación con el proceso proyectual arquitectónico, es decir, en su relación con la construcción de una metodología de diseño, dentro de la cual la práctica opera como fundamento que sostiene y valida la producción del objeto arquitectónico. Vinculado con ello está el problema de la naturalización del método de prueba y error, instalado en la FAU por fuerza de la tradición y transmitido por los profesores. Los ruidos en la comunicación y el predominio de prácticas individualistas de aprendizaje son otros de los problemas que operan como obstáculos para aprender.

En lo concerniente a la enseñanza, se alude a las contradicciones entre teorías profesadas y teorías al uso,

3 Las representaciones son "una manera de interpretar y de pensar nuestra realidad cotidiana, una forma de conocimiento social... la actividad mental desplegada por individuos y grupos a fin de fijar su posición en relación con situaciones, acontecimientos, objetos y comunicaciones que les conciernen". JODELET (2008) La representación social: fenómenos, conceptos y teoría. Paidós, Bs. As.

4 WOLFGANG JONAS. A Scenario for Design, s/fecha. Disponible en URL: http://echo.iat.sfu.ca/library/ Jonas_01_scenario.pdf. 
"... matriz formativa que heredamos y que reproducimos, nos queda repensar rigurosamente cuáles son los aspectos que consideramos positivos y conviene mantener..."

así como al tipo de formación requerida para el ejercicio profesional.

En esta oportunidad nos referiremos a la relación teoría-práctica y los conocimientos previos, visualizados desde una lectura de las trayectorias de los estudiantes.

\section{HALLAZGOS}

\section{Las trayectorias de los estudiantes. Insuficien- cia de los conocimientos previos para la forma- ción profesional}

Los desarrollos de la didáctica muestran que no es posible ni deseable establecer un método único para la enseñanza, cualquiera sea la disciplina de que se trate. Al mismo tiempo, esta disciplina ofrece herramientas para construir respuestas a la infinidad de problemas que plantea la enseñanza. En este punto se enuncian y describen algunas cuestiones inherentes a las trayectorias de los estudiantes, que deben ser consideradas a la hora de pensar las estrategias didácticas para la enseñanza del diseño proyectual, con lo que no ignoramos el hecho de que, en la realidad, trayectorias y enseñanza operan dialécticamente.

Se encuestó al $50 \%$ de los 67 inscriptos en el TFC, en el inicio de la cursada, en 2013. Resulta relevante el hecho de que solo el $35 \%$ de los estudiantes encuestados no tiene antecedentes laborales. De entre los que trabajan, algunos desarrollan más de una actividad; el $20 \%$ se desempeña en una empresa constructora, y el $5 \%$ en alguna dependencia del Estado, pero en calidad de becario/a; el resto de los encuestados es trabajador/a independiente. El $25 \%$, trabaja para un profesional, el $10 \%$, como empleado de comercio, el $5 \%$ como profesor/a particular de materias técnicas, el $5 \%$ en algún rubro de la construcción y el $5 \%$ restante como técnico de PC e instalador de redes.
Estos porcentajes muestran la importancia de que la facultad asuma la responsabilidad de ofrecer los conocimientos necesarios para construir el capital cultural requerido por la universidad, aun cuando se esté autorizado a suponer que esos conocimientos fueron adquiridos en la educación secundaria. Los datos relativos a la educación secundaria de los encuestados refuerzan el planteo anterior. El $25 \%$ posee título de Maestro Mayor de Obras, un $10 \%$ cuenta con título de Técnico Eléctrico o Electromecánico, el $15 \%$ egresó como Bachiller, el $5 \%$ posee título de Perito Mercantil o de Administrativo Contable, el 15 \% egresó de la educación Polimodal con la modalidad Economía y Gestión de las Organizaciones y otro $15 \%$, con la modalidad Humanidades y Ciencias Sociales, mientras un $5 \%$ es Bachiller con Orientación Docente.

Al respecto, cabe señalar que aquellos que poseen formación técnica no han accedido a una educación que contemple el campo de las ciencias humanas, sobre el que gravita gran parte de la teoría del diseño. Mientras tanto, aquellos con trayectoria en ese campo, egresaron de escuelas secundarias bajo el régimen de la Ley Federal de Educación, cuyo impacto ha sido larga y profundamente analizado en términos de fracaso. Aludimos a estas cuestiones porque el aprendizaje está siempre estrechamente vinculado con la construcción, por parte de los estudiantes, de determinados conocimientos previos. Si bien se denominan conocimientos previos tanto a los que provienen de experiencias de vida, como a los que provienen de la experiencia educativa formal de los estudiantes, nos interesan los últimos, pues solo sobre la base de un andamiaje conceptual será posible hacer del taller un espacio en que se logren síntesis, exigidas para una adecuada formación profesional.

En la práctica cotidiana se advierte que los estudiantes inician el cursado de la asignatura sin el andamiaje necesario para integrar los conocimientos de las materias que se ocupan del estudio de estructuras, historia, sociología, construcciones e instalaciones en un 
"La pretensión es sentar bases para el ejercicio de la profesión, mediante el manejo adecuado de los conocimientos que entran en juego en ella."

todo que devendrá, si son capaces de hacerlos operar coherentemente, en un objeto construible, habitable, estéticamente valioso y adecuado a las demandas de quienes lo habitarán. Ello sin dejar de considerar las demandas objetivas de un medio social más amplio que, como tal, trasciende a los individuos y sus intereses particulares cuando estos entran en conflicto con las necesidades objetivas de la comunidad. Al respecto, ante la pregunta "¿Qué metodología emplea para el diseño arquitectónico?", la totalidad de los estudiantes encuestados no menciona metodología de diseño alguno. Como respuesta, describen secuencias de tareas que realizan durante el proceso proyectual y herramientas empleadas para expresar gráficamente la idea. Esto muestra la escasa apropiación de algún método, así como la imposibilidad de distinguir entre método, proceso y herramientas:

"Información-análisis-bosquejos-croquis-síntesis-anteproyecto.

Información: del tema, casos, obras ejecutadas, proyectos etc. Reglamentación.

Análisis: Condicionantes y determinantes, análisis de ejemplos. Aplicación al caso.

Bosquejos-Croquis: a nivel urbano y de lote, terreno. Graficación de ideas resultantes.

Síntesis: de la idea. Dibujos técnicos y asistidos. Resolución de tecnología.

Anteproyecto: graficación técnica normativa. Detalles constructivos, de Instalaciones, etc." (E1).

"Análisis, investigación, el tema y revisar ejemplos".

"La metodología empleada para un diseño arquitectónico sería primero analizar el problema de diseño que se nos presenta, comparar con casos similares y sacar nuevas conclusiones para un buen planteamiento del 'problema diseño' y con ello determinar un marco de referencia para determinar condicionantes y determinantes para partir de idea generadora y trabajar de manera gráfica". (E 17)
Algunas veces, son los mismos estudiantes quienes expresan demandas de formación coherentes con lo que enunciamos precedentemente:

“... Faltaría aplicar un poco más las teorías que se dan en 'Desarrollo Urbano' y 'Sociología Urbana'. Y también algún apoyo en instalaciones especiales propios de la Complejidad de los proyectos de T.F.C. y Construcciones complementarias propias de la temática". (E 25)

Como TFC es el último taller, es legítimo suponer que los estudiantes que inician el cursado llegan habiendo construido una metodología de diseño producto de aprendizajes logrados en las asignaturas de niveles anteriores. Se trata de aprendizajes referidos a la resolución de problemas funcionales, compositivos y morfológicos, tecnológico-constructivos y estructurales. En esta asignatura los objetivos se dirigen a la realización, por parte de los estudiantes, de experiencias de formación orientadas a la adquisición de destrezas para resolver ámbitos de actividades humanas públicas y privadas. La pretensión es sentar bases para el ejercicio de la profesión, mediante el manejo adecuado de los conocimientos que entran en juego en ella. Esto también se pone de manifiesto cuando los estudiantes reconocen la importancia del interjuego de los distintos campos del conocimiento en el diseño arquitectónico; y así lo explicitan frente a la pregunta "¿Cuáles son los campos del conocimiento en los que cree necesita más apoyo del equipo docente?". El 50 $\%$ de los encuestados dice necesitar apoyo durante el proceso de diseño proyectual:

"... en el proceso proyectual, para lograr un buen trabajo". (E 12)

"Necesitaría saber cómo arrancar y continuar un diseño con menos dudas, es decir tener un buen proceso proyectual...". (E 6)

"Quiero que me guíen durante el proceso que lleva 
el proyecto... para saber cómo trabajar cuando me reciba". (E 2)

El $25 \%$ cree necesitar reforzar saberes sobre urbanismo. El $40 \%$ requiere reforzar conocimientos de tecnología, instalaciones, construcciones y estructuras, y el $15 \%$ dice necesitar aportes de morfología:

"Necesito afianzar conocimientos en lo que respecta al área tecnológica, instalaciones especiales para edificios complejos. Composición funcional y morfológica de obras de gran envergadura... mayores conocimientos para hacer proyectos de urbanismo o intervención territorial". (E 7)

"Necesito más apoyo en el tema urbanismo...". (E 30)

"Instalaciones especiales como por ejemplo, instalaciones contra incendios. Intervención urbana". (E 28)

"En la parte tecnológica, instalaciones, estructuras y en conocimientos necesarios para hacer un proyecto de intervención urbana...". (E 4)

"Los campos en los que necesito más apoyo son: en instalaciones, construcción y cómputo". (E 1)

"Conocimientos técnicos-constructivos, estructuras... Aspectos morfológicos". (E 30)

Hay un $20 \%$, que confunde campos del conocimiento con situaciones problemáticas, aunque aparece claro el paralelo que se establece entre la complejidad de la realidad y la del diseño:

"En realizar temas que tengan que ver más con la realidad. Clases teóricas relacionadas con los temas a desarrollar". (E 15)

"Justamente en desarrollar temas acordes a la realidad... aprender a resolverlos antes de salir de la facultad". (E 5)
Evidentemente, hay aquí, como en toda profesión, un entrelazamiento de variables, muchas veces difíciles de conjugar en la práctica del diseño, pues se requiere la toma de conciencia acerca de cuál sería el fin último de un objeto arquitectónico cualquiera, desde un posicionamiento de la práctica profesional como práctica de servicio a la sociedad.

Aparece también aquí la cuestión del uso del lenguaje en la práctica arquitectónica, que merece ser analizado desde dos dimensiones de la práctica: la práctica gráfica y la práctica discursiva. Básicamente, la arquitectura se transmite por medio de imágenes, que no deberían estar divorciadas del marco teórico desde el cual se las concibe, considerando el contexto desde el cual se las formula. De no ser así, el objeto diseñado carece de la coherencia necesaria entre obra diseñada y fundamentos que la sostienen y justifican. Porque la arquitectura, además de ser mostrable mediante imágenes, debe ser factible de explicarse, desde los supuestos teóricos de las ciencias en que se funda.

La palabra o el discurso es así el medio a través del cual se objetivan las representaciones. Según VASILACHIS (1998: 301), las representaciones son "construcciones simbólicas individuales o colectivas a las que los sujetos apelan o las que crean para interpretar el mundo, para reflexionar sobre su propia situación y la de los demás y para determinar el alcance y la posibilidad de su acción histórica". Sin embargo, en el trabajo áulico, es bastante usual que los estudiantes elaboren proyectos de arquitectura que, aun cuando respeten reglas compositivas, respondan a imágenes preconcebidas y ajenas al contexto para el que fueron pensadas.

La enseñanza se orienta, pues, a la formación de los estudiantes. Entendemos la formación en el sentido que le confiere FERRY (1997), cuando dice que formarse es "(...) adquirir una cierta forma. Una forma para actuar, para reflexionar y perfeccionar esta forma" (pág. 53). FERRY distingue la formación de la enseñanza y el aprendizaje. La enseñanza y el aprendizaje pueden entrar en la formación, como soportes, 
"las disciplinas constituyen un sistema de control en la producción de discurso, fijando sus límites por medio de la acción de una identidad que adopta la forma de una permanente reactivación de las reglas".

pero la formación como desarrollo personal consiste en "encontrar formas para cumplir con ciertas tareas para ejercer un oficio, una profesión, un trabajo" (FERRY, 1997). Pero además, como dice FILLOUX (1997: 42), "(...) formación es deformarse, reformarse, es encontrar nuevas formas".

Todo proceso de formación está determinado por la relación profesor-alumno; puesto que la "...formación es un diálogo entre personas (...) capaces de realizar un retorno sobre sí mismo porque no se puede tomar conciencia de lo que soy sino por intermedio de lo que el otro me devuelve a mí (...) Es a través del conocimiento del otro como sujeto que yo puedo reconocerme como sujeto" (FILLOUX, 1996: 37). En el proceso de formación, se modifica la subjetividad misma en tanto ella se constituye en una relación dialéctica entre lo material y lo simbólico, lo individual y lo colectivo, todo ello mediatizado por las instituciones. Paralelamente, se modifica también el sentido y el fin último de la formación para el ejercicio profesional, pues entran en conflicto los valores que la guían y las prácticas que devienen de ellos. La formación nos habla de una transformación que opera sobre la concepción del mundo, a través de la ruptura de prejuicios, preconceptos naturalizados y prácticas sociales determinadas por el contexto.

\section{Dicotomía teoría-práctica}

Como planteamos anteriormente, en la FAU-UNNE, por fuerza de la tradición, el TA se concibe como materia teórico-práctica. El señalamiento de la doble dimensión de los conocimientos (teóricos y prácticos) deviene de una concepción que designa y clasifica a las asignaturas como teóricas por un lado y prácticas por el otro. A su vez, esta tradición se funda en una concepción positivista de la ciencia que opone objeto a sujeto en el acto de pensar, y opone así el pensar reflexivo a la acción. La perspectiva crítica de la enseñanza permite superar —entre otras - la dicotomía teoría-práctica ${ }^{5}$, para lo cual es fundamental el esfuerzo del docente, lo que supone a la vez reconocer que la organización disciplinar para la enseñanza es un requisito para producir conocimiento, pero no una característica de la realidad.

PIAGET (1975) postula el conocimiento como construcción social. Desde la perspectiva constructivista, el desarrollo cognoscitivo es complejo, y no consiste simplemente en adquisición de respuestas, sino en un complejo proceso constructivo en el que el sujeto opera activamente con su entorno (incluidos otros sujetos), y a la vez que construye conocimientos, edifica también las propias estructuras intelectuales o cognitivas. A través de las estructuras opera: el sujeto elabora esquemas con los cuales interpreta el mundo y actúa. Los esquemas ofrecen una imagen simplificada, que representa lo que puede repetirse y generalizarse en una acción. Cuando los esquemas son rígidos, lejos de aproximar al conocimiento de la realidad, pueden constituir lo que GASTÓN BACHELARD (1993) denomina obstáculos epistemológicos, que obturan el aprendizaje.

Así pues, como muestra BACHELARD, no todo conocimiento previo es de por sí valioso para construir conocimientos. Su valor radica, en el caso que nos ocupa, en su validez científica y su significatividad. La importancia de estos conocimientos es tal, que AUSUBEL (1968: 11) afirma que "De todos los factores que influyen en el aprendizaje el más importante consiste en lo que el alumno ya sabe. Averígüese esto y enséñese consecuentemente".

La experiencia de enseñanza en la asignatura indica, efectivamente, que una de las dificultades relacionadas con la construcción de la metodología de diseño

\footnotetext{
5 "Desde una perspectiva crítica, el profesor necesita desarrollar una comprensión sistemática de las condiciones que conforman, limitan, y determinan la acción de modo que tales restricciones puedan ser tenidas en cuenta. Y esto requiere la participación activa de los prácticos en la formulación y articulación colaborativa de las teorías inminentes en sus propias prácticas, y el desarrollo de esas teorías mediante la continua acción y reflexión" (CARR Y KEMMIS, 1983, p. 149 en J. Contreras, Enseñanza, Currículum y Profesorado: introducción crítica a la didáctica. Madrid: Akal.
} 
radica en que un alto porcentaje de los estudiantes inician la cursada con escasos conocimientos necesarios para andamiar otros nuevos. AUSUBEL señala la necesidad de identificar los conocimientos previos de los estudiantes, que resultan relevantes para lo que queremos enseñar; se trata de "conceptos inclusores", sobre los cuales trabajar para que los estudiantes puedan indagar en sus esquemas y estar preparados para su revisión.

Con respecto al distanciamiento entre teoría y práctica DA CUNHA (200?: 24) sostiene por un lado que este ha servido siempre para desvincular el quehacer humano del conocimiento científico y el saber académico, y que el sustento de que "la teoría puede ser encajada en la práctica" se funda en el paradigma positivista de la ciencia y el propio transcurso histórico de la universidad, y desde esta mirada la teoría siempre precede a la apropiación del objeto de conocimiento.

Esto proviene de la organización académica del conocimiento (universitario y áulico), en la que subyace la creencia de que el estudiante debe primero conocer la teoría para ser capaz, luego, de interpretar la realidad. El peso de tal ruptura teoría-práctica en la enseñanza del diseño arquitectónico deriva en proyectos que no responden a sus fines.

Contrariamente a esta tradición, la formación de profesionales técnicamente competentes consistiría básicamente en un proceso dialéctico de la articulación teoría-práctica: "la teoría supone práctica teórica, la práctica puede constituirse en motivo de teorización" (MASTACHE, 2007: 84). Los aportes de MASTACHE resultan significativos para pensar la articulación teoría-práctica, "concebida, más que como un momento de aplicación de la teoría, como una instancia que requiere movilizar todo lo que se sabe, adquirir nuevos conocimientos o dar origen a nuevas conceptualizaciones teóricas. Y la teoría es percibida, más que como un discurso científico a ser estudiado, como una herramienta a ser adquirida y utilizada para dar respuesta a situaciones concretas - analizar, diagnosticar, resolver, proyectar-" (MASTACHE, 2007: 84). Ello implica romper con la distancia tradicional que ubica a la teoría como un constructo discursivo, y a la práctica como acción sin teoría, o como mucho, aplicación de esta. La articulación teoría-práctica se vuelve así una cuestión fundamental en el proceso de enseñanza aprendizaje en el TA. Posicionados desde una concepción crítica de la enseñanza, no podemos sino reconocer su indisoluble relación dialéctica, en la cual hechos y situaciones están en constante contradicción y la práctica es teoría en la acción.

La casi totalidad de los estudiantes encuestados, más precisamente el $92 \%$, da cuenta de la importancia de teorizar sobre la práctica, como lo demuestran sus propios dichos:

"Creo que es bueno que me corrijan haciéndome pensar en los conceptos... y por qué hago este proyecto de esta manera, en vez de otro". (E 15)

“... porque es mejor que nos hagan analizar, nos hagan comparar con obras de la historia y de nuestra región... y no que me digan cambiá esta puerta o sacá este dormitorio... o llevá para allá este espacio... así pensamos y relacionamos los proyectos con la teoría". (E 22)

"... eso siempre exige un porqué para explicar los proyectos, y hay que tener argumentos para poder hacer los proyectos y poder llevarlos adelante... sino parece que se proyecta por proyectar, sin fundamento". (E 20)

"Es más difícil pensar en la teoría sobre la práctica del proyecto... pero creo que es mejor, me parece como más seguro... porque al tener sentido lo que se hace, pierde eso que parece como de casualidad". 
"En pleno siglo XXI, nos encontramos con los mismos problemas, objetos de discusión de modernistas y posmodernistas sobre qué es la arquitectura y cómo se enseña."

\section{REFLEXIONES FINALES}

El abordaje de la enseñanza del diseño arquitectónico exige considerar dos campos del conocimiento: el diseño arquitectónico y las prácticas de enseñanza. A la vez, ambos campos de desempeño profesional (del arquitecto y del docente) suponen un posicionamiento epistemológico, desde el cual pensar y actuar.

Desde su desarrollo hasta el momento es innegable el aporte de las escuelas de arquitectura Bauhaus, UIm y las experiencias de enseñanza de KAHN, que discutieron, entre otras cosas, acerca de los contenidos disciplinares que enseñar y cómo enseñarlos. Enseñar el método de diseño implica, entre otras cosas, colaborar con los estudiantes en la construcción de una metodología de abordaje para resolver problemáticas urbano-arquitectónicas, y en la recuperación de conocimientos y experiencias previas. El sujeto construye conocimientos en interacción con el contexto. La función del enseñante es mediar entre el contenido y los estudiantes, proporcionando las ayudas o andamios necesarios para que estos avancen progresivamente en la apropiación significativa de los saberes.

Como sostiene BRUNER (1988), la labor docente cobra especial importancia con respecto al conocimiento disciplinar del profesor para decidir lo que es epistemológicamente relevante, con respecto a su conocimiento metodológico para enseñar el contenido específico, su capacidad para interpretar intereses y necesidades de los estudiantes relacionadas con requerimientos del contexto socioeconómico, histórico y político. El planteo de BRUNER nos invita a considerar la incidencia de nuestras propias trayectorias de formación en las prácticas de enseñanza.

Pensar en la enseñanza exige, por supuesto, considerar diversas cuestiones que hacen a la práctica, de las cuales no siempre somos conscientes los docentes. En primer lugar, en toda práctica subyace una concepción del currículo. En segundo lugar, la enseñan- za es siempre un problema que encierra cuestiones epistemológicas, es decir, una concepción del conocimiento, de la verdad y de la forma de transmitirlo. En estrecha relación con esto último, está la cuestión de la relación entre la enseñanza y los modos de apropiación del conocimiento por parte de los estudiantes, lo que involucra la propuesta didáctica, que incluye, entre otras cosas, las estrategias de enseñanza.

Por otra parte, la perspectiva crítica rompe con los esquemas tradicionales de los enfoques funcionalistas en sociología de la educación, y visualiza la educación en su carácter social y ético-político, al advertir el interjuego de relaciones dialécticas a través de las cuales se organiza lo social. El reconocimiento de la dialéctica social obliga, asimismo, a un posicionamiento ético-político del profesional de la arquitectura y del docente. Al respecto, FIORENZA (1987: 2) sostiene: "se puede o no con-dividir la poética formal, pero es evidente el rigor metodológico y la honestidad proyectual. Dos elementos no siempre presentes en la arquitectura actual, que atraviesa por un período de crisis de contenidos culturales y está enferma de formalismo y "post-modernismo".

En pleno siglo $\mathrm{XXI}$, nos encontramos con los mismos problemas, objetos de discusión de modernistas y posmodernistas sobre qué es la arquitectura y cómo se enseña. La persistencia de dichos problemas constituye el trasfondo del núcleo de dificultades propias de la transmisión del conocimiento en el ámbito académico, lo que indica, a la vez, la necesidad de repensar las estrategias de transmisión del conocimiento.

Una cuestión que no se debe perder de vista en la construcción del conocimiento es el hecho de que este debe ser "leído" en el marco de las trayectorias estudiantiles, lo que vuelve a poner en juego la relación entre lo social y lo individual con el conocimiento. Dada la imposibilidad de construcción de la nada, se hace necesario conocer la producción teórica ofrecida por la historia de la arquitectura sobre la cuestión 
"Desde este lugar, para quienes eligen la carrera de Arquitectura es importante aprender a liberar la mirada, a ser atentos y no solo a estar atentos."

del diseño, de lo que resulta obvio que no hay una única metodología de diseño de validez universal. Por el contrario, se trata de que cada estudiante sea capaz de apropiarse de conocimientos y estrategias valiosos para la construcción de caminos propios hacia el diseño de objetos materializables.

Desde la perspectiva práctico-reflexiva del currículum, la planificación de la enseñanza contribuye más que a solucionar problemas a que el profesor pueda detectarlos, descubrirlos y abordarlos; en ese sentido, oficia como hipótesis de trabajo que se pone a prueba (STENHOUSE, 1985). Como expresa ELLIOTT (1991: 72), la enseñanza se concibe como una forma de investigación encaminada a comprender cómo traducir los valores educativos a formas concretas de práctica.

En el desarrollo del currículum, se pone a aprueba el conocimiento disponible y se aprende de la práctica para mejorarlo en un proceso continuo de reconstrucción del conocimiento. Pero la práctica no es un bien exclusivo de los docentes, pues un currículum no es un conjunto de imposiciones sobre los estudiantes, sino más bien un conjunto de posibilidades y de esperanzas prácticas enmarcadas en parte por los estudiantes. Todos aprendemos, "nadie no sabe nada" dice HAMILTON (STENHOUSE, 200?: 208), puesto que tanto estudiantes como profesores constituyen un complejo social interactivo: siempre aprenden algo, porque el aula, como medio ambiente de aprendizaje, no es un marco preordenado, sino socialmente construido.

En el mismo sentido, podemos hablar de la construcción de conocimientos sobre lo que hacemos cuando enseñamos. El proceso de reflexión sobre las prácticas, movido por razones prácticas y teóricas, se justifica política y éticamente, en tanto encierra una intención y supone un deber ser. En última instancia, tal empresa depende de la calidad de los profesores. La profesión docente se asienta sobre un doble juego de diferenciación e integración de saberes instrumentales, éticos y políticos, y focaliza los dispositivos de reflexión como eje de articulación. Debe ser capaz de mejorar la calidad de la educación a través de una práctica pedagógica reflexiva: "la idea de los docentes como profesionales sólo tiene sentido en un contexto en el que se considere la educación como una práctica social" (ELLIOTT, 1991).

Nos cabe entonces pensar la profesión ya no desde una perspectiva colectiva de resolución de problemas y de atención de necesidades específicas de las personas. Debemos pensarla en un marco más amplio de reflexión sobre el significado de las prácticas profesionales para una sociedad más justa, susceptible de ser construida en la medida en que los sujetos que transitan por nuestras aulas aprendan a pensar y actuar en forma autónoma y solidaria.

Con respecto a los conocimientos requeridos para el ejercicio profesional, MASSCHELAIN (2006) habla de la necesidad de "educar la mirada" y de "La necesidad de una pedagogía pobre". "Educar la mirada no es alcanzar una mirada liberada o crítica (...), sino liberar nuestra propia mirada. No se trata de lograr mayor conciencia o de estar más alerta sino de volvernos atentos, de prestar atención (...) Estar atento es el estado mental en que el sujeto y el objeto juegan el mismo juego. Es un estado mental que se abre al mundo de modo que el mundo se 'presentifique' delante de mí y (que 'llego' a ver) que puede transformarme". Desde este lugar, para quienes eligen la carrera de Arquitectura es importante aprender a liberar la mirada, a ser atentos y no solo a estar atentos. En el ejercicio de la profesión, ser atentos significa ser un sujeto emancipado de los encorsetamientos teóricos para que el objeto nos hable. Significa también emanciparse de las prácticas dominantes, de la actitud de poseedor del saber o del simple mercader, dejando que nos hablen los otros, las nuevas subjetividades y las nuevas y emergentes racionalidades y expresiones estéticas.

Estas nociones entran en conflicto con lo aprendido acerca de que educarse era "alcanzar el conocimien- 
"Esperar que los alumnos sean capaces de apropiarse de saberes significativos es legítimo solo cuando nos mueve la voluntad de ser los primeros en apropiarnos de los mismos saberes y de aquellos que explican la compleja relación entre el enseñar y el aprender."

to" y acumularlo, y su reemplazo por la búsqueda de diálogo entre sujeto y objeto. Así, la pedagogía pobre es más difícil de perseguir. Estas nociones se entrelazan con la idea de FREÜD, recuperada por ENRIQUEZ (2002), de que "hay profesiones en las que estamos seguros de que, hagamos lo que hiciéremos, siempre vamos a tener resultados insuficientes, son las que podríamos llamar las profesiones imposibles $u$ oficios y entre las que las dos más conocidas son el arte de enseñar y el arte de gobernar" (pág. 23). Esta es, en última instancia, la problemática que abordan quienes se encuadran en posiciones críticas, porque lo complejo de nuestra realidad se transfiere también al aula, pues siempre aparecen rasgos del mundo del trabajo, en los que se entremezclan cuestiones culturales y socio-históricas: "... no resulta fácil trabajar en nuestra sociedad calificada como globalizada, sobremoderna o posmoderna" (SAGASTIZÁBALE ET ÁL., 2006: 23), puesto que los cambios sociales se evidencian con más fuerza en el momento de enseñar $y$ de aprender.

Desde estos presupuestos podemos intentar responder algunos de los interrogantes sobre el quehacer docente: ¿cómo enseñar un método de diseño en la práctica proyectual?, ¿quiénes son los estudiantes a los que les enseñamos?; ¿qué valor otorgar a los conocimientos y experiencias acumuladas en las distintas trayectorias?; ¿qué estrategias resultan adecuadas para la construcción de conocimientos?; ¿cómo conducir a los estudiantes a la comprensión de que el quehacer profesional requiere una relación solidaria entre teoría y práctica?

Esperar que los alumnos sean capaces de apropiarse de saberes significativos es legítimo solo cuando nos mueve la voluntad de ser los primeros en apropiarnos de los mismos saberes y de aquellos que explican la compleja relación entre el enseñar y el aprender. DAY (2005: 47) habla de la reflexión en relación con la acción, la que supone una postura crítica más general e involucra cuestiones éticas, políticas e instrumenta- les que se encuentran en el pensamiento y práctica docente. Así concebida, la reflexión permite revisar las decisiones que se toman, y comprender las interrelaciones entre fines, prácticas y contextos normativos de la enseñanza. El planteo de DAY constituye el telón de fondo desde el cual la reflexión sobre la práctica y la redefinición de las estrategias adquieren sentido, pues como el mismo DAY nos dice, "(...) los profesores que reflexionan en, sobre y en relación con la acción, se inscriben en una investigación que intenta al mismo tiempo, comprenderse mejor a sí mismos como docentes y mejorar su labor profesional" (DAY, 2005).

La reflexión sobre la enseñanza del diseño arquitectónico tensiona las prácticas instaladas por fuerza de la tradición en la facultad y la interpela en clave social, hacia la búsqueda de espacios de diálogo entre los saberes docentes y las trayectorias socioeducativas de los estudiantes. Como muestran las opiniones de los estudiantes, para el mejoramiento de la enseñanza se requieren espacios institucionales de articulación, que posibiliten, como plantea AGUERRONDO (2009), construir para el conjunto de los estudiantes continuidades pedagógico-didácticas, que den forma a la experiencia de aprendizaje a lo largo de sus trayectorias educativas en la universidad.

En este sentido, resulta fundamental el trabajo colectivo de los docentes, requisito indispensable de la calidad educativa, entendida en su estrecha relación con la democratización del conocimiento, asumida, a la vez, como responsabilidad institucional y no como mera responsabilidad individual de cada estudiante, lo que los dejaría librados a las determinaciones sociales que pesan sobre ellos.

La no obligatoriedad de la educación universitaria no nos exime, sin embargo, de la obligación de ensayar respuestas institucionales para acompañar y fortalecer las trayectorias estudiantiles, atendiendo a los principios de inclusión, igualdad y justicia social que regulan hoy las políticas públicas tendientes al reco- 
nocimiento efectivo de los derechos fundamentales de las personas, entre los que está el derecho a la educación.

Un primer paso para la construcción de acuerdos colectivos podría ser la organización de espacios institucionales para la articulación (intercátedras, departamentales, por áreas y niveles, etc.) y la incorporación definitiva de la enseñanza como tema de la agenda de nuestra facultad.

\section{BIBLIOGRAFIAA}

AGUERRONDO, Inés (2009) Conocimiento complejo y competencias educativas. IIPE/UNESCO Sede Buenos Aires UNESCO.

AUSUBEL - NOVAK - HANESIAN (1983) Psicología Educativa: Un punto de vista cognoscitivo. (2. ${ }^{a}$ Ed.). Trillas. México.

BACHELARD, Gastón (1993) La formación del espíritu científico. Contribución a un psicoanálisis del conocimiento objetivo. (19. ${ }^{a}$ Ed.). Siglo XXI Editores. Traducción de José Babini, México.

BRUNER, Jerome (1988) Realidad mental y mundos posibles. España. Gedisa, Barcelona.

CONTRERAS Domingo, José (1990). Enseñanza, currículum y profesorado: introducción crítica a la didáctica. Akal, Madrid.

DA CUHNA, María Isabel (200?) Universidad futurante: producción de la enseñanza e innovación. Facultad de Filosofía y Letras, UBA, Buenos Aires.

DAY, Christopher (2005) Formar docentes: cómo, cuándo y en qué condiciones aprende el profesorado. Narcea, Madrid.

ELLIOTT, John (1991) El cambio educativo desde la investigación. Morata, Madrid.

ENRIQUEZ, E. (2002) La institución y las organizaciones en la educación y la formación. Ediciones Novedades Educativas, Facultad de Filosofía y Letras, UBA, Buenos Aires.

FERRY, Giles (1990) I trayecto de la formación. Los enseñantes entre la teoría y la práctica. Paidós, Buenos Aires.

FERRY, Giles (1997) Pedagogía de la Formación. Ediciones Novedades Educativas, Buenos Aires.

FILLOUX, Jean Claude (1996) Intersubjetividad y formación. El retorno sobre sí mismo. Ediciones Novedades Educativas, Buenos Aires.

FIORENZA, Dante A. (1987) Bruno Morassutti: Arquitectura Moderna, a/mbiente, N. ${ }^{\circ}$ 55. CEPA, Buenos Aires.

FOGAR, Mariela del Carmen, RODRÍGUEZ, Carmen Isabel y MAUREL, María del Carmen (2010). Indicadores de equidad en el acceso al conocimiento en la formación docente. Ministerio de Educación de la Nación. INFOD - RED PROPONE.

LEATHERBARROW, David (2014). "Recomenzando la tarea de la investigación proyectual”. En: SUMMA +, N.o 134, Año 21. 88-93.

MALDONADO, Tomás (1979) Técnica y cultura. El debate alemán entre Bismarck y Weimar. Infinito, Milán.

MASTACHE, Anahí. (2007) Formar personas competentes: desarrollo de competencias tecnológicas y psicosociales. Noveduc, Buenos Aires. MASSCHELEINE, J. (2006) Educar la mirada. La necesidad de una pedagogía pobre. Manantial, Buenos Aires.

MELE, Jorge (2011) Relatos críticos de la arquitectura como modo de producción cultural. Sociedad Central de Arquitectos. Nobuko, Bs. As. MUNARI, Bruno (2004) Cómo nacen los objetos. Apuntes para una metodología proyectual. (10. ${ }^{a}$ ed.). G. Gili Diseño, Barcelona.

NOVAK, J. D. (1988) Teoría y práctica de la educación. Editorial Alianza Universidad.

OTL, Aicher (1994) El mundo como proyecto. (4ª Ed.). Gustavo Gili, SA, México.

PIAGET, J. e INHELDER (1975) Psicología del niño. Madrid: Morata.

SAGASTIZABAL, María de los Ángeles; PERLO, Claudia; PIVETTA, Bibiana; SAN MARTín, Patricia S. (2006). Aprender y enseñar en contextos complejos: multiculturalidad, diversidad y fragmentación, pp. 21 - 42 (1. . ed.). Novedades Educativas, Buenos Aires.

SILVA, Carlos Alberto y FOGAR, Mariela del Carmen (2013). "Los estudiantes y su formación. Escenarios y tensiones en la educación técnica. Estudio de casos del Profesorado en Tecnología del INTET y P". En: Actas de Primeras Jornadas Internacionales sobre Conflictos y Problemáticas Sociales y Terceras Jornadas Interdisciplinarias sobre Conflictos y Problemáticas Sociales en la Región del Gran Chaco. UNNE, Facultad de Humanidades, 2, 3 y 4 de mayo de 2013. Publicación en prensa, en: Reviste Theomai. Estudios Críticos sobre Sociedad y Desarrollo. Considerada "Revista Destacada" por los investigadores del CONICET (Encuesta de revistas en Ciencias Sociales, CONICET-Centro Redes).

STENHOUSE, L. (1985) Investigación y desarrollo del currículum. Morata, Madrid.

TIMMLING, Hans Fox (S/F) Reflexiones en torno al proceso de diseño en arquitectura. Recuperado el 6 de junio de 2014, de http://mingaonline.uach.cl/pdf/aus/n5/art02.pdf

WOLFGANG, Jonas (S/F) A Scenario for Design. Recuperado el 24 de nov. de 2013, de http://echo.iat.sfu.ca/library/Jonas01_scenario.pdf 